\title{
Prevalence and Associated Factors of Tinnitus: Data From the Korean National Health and Nutrition Examination Survey 2009-2011
}

\author{
Kyoung Ho Park ${ }^{1}$, Seung Hwan Lee ${ }^{2}$, Ja-Won Koo ${ }^{3}$, Hun Yi Park ${ }^{4}$, Kyu Yup Lee ${ }^{5}$, Young Seok Choi ${ }^{6}$, \\ Kyung Won $\mathrm{Oh}^{7}$, Ari Lee ${ }^{7}$, Ji-Eun $\mathrm{Yang}^{7}$, Sook-Young Woo ${ }^{8}$, Seon Woo Kim${ }^{8}$, and Yang-Sun Cho ${ }^{9}$ \\ ${ }^{1}$ Department of Otorhinolaryngology, Catholic University of Korea, College of Medicine, Seoul, Korea \\ ${ }^{2}$ Department of Otorhinolaryngology, Hanyang University, College of Medicine, Seoul, Korea \\ ${ }^{3}$ Department of Otorhinolaryngology, Seoul National University Bundang Hospital, Seoul National University College of Medicine, Seongnam, Korea \\ ${ }^{4}$ Department of Otorhinolaryngology-Head and Neck Surgery, Ajou University, College of Medicine, Suwon, Korea \\ ${ }^{5}$ Department of Otorhinolaryngology-Head and Neck Surgery, Kyungpook National University, College of Medicine, Daegu, Korea \\ ${ }^{6}$ Department of Otorhinolaryngology-Head and Neck Surgery, Chungpook University, College of Medicine, Chung-ju, Korea \\ ${ }^{7}$ Division of Chronic Disease Surveillance, Korea Centers for Disease Control \& Prevention, Osong, Korea \\ ${ }^{8}$ Biostatistics Team, Samsung Biomedical Research Institute, Seoul, Korea \\ ${ }^{9}$ Department of Otorhinolaryngology-Head and Neck Surgery, Samsung Medical Center, Sungkyunkwan University School of Medicine, Seoul, Korea
}

Received January 29, 2014; accepted April 17, 2014; released online June 21, 2014

Copyright (C) 2014 Kyoung Ho Park et al. This is an open access article distributed under the terms of Creative Commons Attribution License, which permits unrestricted use, distribution, and reproduction in any medium, provided the original author and source are credited.

\section{ABSTRACT}

Background: Tinnitus is a common condition and frequently can be annoying to affected individuals. We investigated the prevalence and associated factors for tinnitus in South Korea using the data from the Korea National Health and Nutrition Examination Surveys (KNHANES) during 2009-2011.

Methods: KNHANES is a cross-sectional survey of the civilian, non-institutionalized population of South Korea $(n=21893)$. A field survey team that included an otolaryngologist moved with a mobile examination unit and performed interviews and physical examinations.

Results: Among the population over 12 years of age, the prevalence of any tinnitus was $19.7 \%$ (95\% CI $18.8 \%-20.6 \%)$. Tinnitus was more prevalent in women, and the prevalence rate increased with age $(P<0.001)$. Among those with any tinnitus, $29.3 \%$ (95\% CI 27.3\%-31.3\%) experienced annoying tinnitus that affected daily life. Annoying tinnitus also increased with age $(P<0.001)$, but no sex difference was demonstrated $(P=0.25)$. In participants aged 40 years or older, age, quality of life, depressive mood, hearing loss, feeling of dizziness, and rhinitis were associated with any tinnitus $(P<0.05)$. Age, hearing loss, history of cardiovascular disease, and stress were associated with annoying tinnitus $(P<0.05)$.

Conclusions: Tinnitus is a common condition, and a large population suffers from annoying tinnitus in South Korea. Public understanding of associated factors might contribute to better management of tinnitus.

Key words: tinnitus; epidemiology; associated factor; South Korea

\section{INTRODUCTION}

Tinnitus, the perception of sound from the ears or head without an audible external source, is a symptom, rather than a disease entity, that originates internally without an external auditory input. It is relatively common in the elderly, but the actual reported prevalence varies according to the surveyed region and definition of tinnitus. ${ }^{1,2}$ For example, the prevalence of tinnitus was reported as $5.17 \%$ in a study from Egypt $^{3}$ and $25.3 \%$ in a report from the 1999-2004 National Health and Nutritional Examination Survey (NHANES) in the United States (US). ${ }^{4}$

Nation-wide epidemiologic studies that are conducted by government organizations can provide powerful data for investigating the national prevalence of disease conditions. The Korea National Health and Nutrition Examination Survey (KNHANES) was started in 1998 to examine the general health and nutrition status of populations in South Korea. 
From 2009 to 2011, a total of 10000 to 12000 individuals were selected annually, and the participating household members were interviewed on their health and nutrition and asked to undergo a basic health assessment that included blood pressure measurements, blood and urine collection, a pulmonary function test, a dental examination, an ophthalmologic examination, and an otolaryngologic examination. In the otolaryngologic interview and examination, history of tinnitus was surveyed and otologic evaluations of the tympanic membrane (TM), hearing, and balance were conducted in participants of appropriate ages.

The present study was undertaken to determine the national prevalence of tinnitus in South Korea based on survey data obtained from the 2009 to 2011 KNHANES and to investigate associated factors. Identification and modification of these factors may aid in reducing the incidence or severity of tinnitus and, in turn, facilitate the efficient allocation of public health resources aimed at reducing the negative effects of tinnitus in everyday life.

\section{MATERIALS AND METHODS}

\section{Study population and data collection}

The KNHANES is an ongoing cross-sectional survey of the civilian non-institutionalized population of South Korea. A field survey team that included an otolaryngologist, an ophthalmologist, and nurse examiners for health assessment moved with a mobile examination unit and performed interviews and physical examinations. Every year, 10000 to 12000 individuals in about 4600 households are selected from a panel to represent the population by using a multistage clustered and stratified random sampling method that is based on the National Census Data. The participation rate of selected households in the past several cycles of KNHANES has been high, ranging from $79 \%$ to $84 \%$. A total of 21893 individuals, representing the 41900788 individuals in South Korea $\geq 12$ years of age, participated in the survey from July 2009 to December 2011. Among them, 9653 male participants represented 20923340 Korean men and 12240 female participants represented 20977448 women.

Written informed consent was obtained from all participants prior to the survey, and approval for this research was obtained from the Institutional Review Board of the Samsung Medical Center (IRB No. 2013-02-031).

\section{Survey for tinnitus and otologic examination}

Participants were asked about their experiences of tinnitus. In response to the questionnaire item "Within the past year, did you ever hear a sound (buzzing, hissing, ringing, humming, roaring, machinery noise) originating in your ear?", examiners were instructed to record 'yes' if a participant reported hearing an odd or unusual noise at any time in the past year. Participants who responded positively to this question were then queried concerning the resulting annoyance in their lives by the following questions: "How severe is this noise in daily life?" (not annoying/annoying (irritating)/severely annoying and causes sleep problem). The participants were grouped as having annoying tinnitus if the severity of tinnitus was annoying or severely annoying.

To determine the prevalence of TM perforation and cholesteatomatous conditions, including retraction pocket and otitis media with effusion (OME), an ear examination was then conducted with a $4-\mathrm{mm} 0^{\circ}$-angled rigid endoscope attached to a CCD camera for all participants $\geq 4$-years-of-age.

The pure tone air-conduction threshold was measured in a sound-proof booth using an automatic audiometer (GSI SA-203; Entomed Diagnostics AB, Lena Nodin, Sweden). Hearing loss was defined as over 25 decibels hearing level ( $\mathrm{dB}$ $\mathrm{HL}$ ) with average air-conduction hearing thresholds measured at $0.5,1,2$, and $3 \mathrm{kHz}$ according to the recommendation from the American Academy of Otolaryngology-Head and Neck Surgery. ${ }^{5}$

\section{Balance survey}

Participants aged 40 years or older were asked whether they had any experience during the prior 12 months of dizziness or imbalance, subjective positional dizziness, or falls in the absence of external factors. To assess balance, participants were asked to stand on a firm surface with their feet approximately $10 \mathrm{~cm}$ apart for at least 15 seconds with their arms crossed, while bending their knees or moving their body to maintain balance. They were not allowed to move their feet with their eyes open (condition 1) or closed (condition 2). Next, we assessed the effect of eliminating somatosensory input on postural stability, by repeating the same procedure but on a foam pad with eyes open (condition 3) or closed (condition 4) for at least 20 seconds. The balance of an individual was scored on a pass/fail basis. A participant failed the balance test if they moved their feet, unfolded their hands, opened their eyes or required the operator to intervene to maintain their balance. As condition 4 evaluated the vestibular function to maintain balance, participants were concluded to have vestibular dysfunction if they did not pass test condition 4 , even if they had passed test conditions $1-3$.

\section{Rhinologic survey and examination}

Participants were interviewed and examined for their nasal symptoms and disorders. The diagnosis of rhinitis was made when the participants had subjective symptoms of watery rhinorrhea, sneezing, itching, and nasal obstruction without a fever or a sore throat within the past year ("Within the past year, did you ever experience symptoms of rhinitis such as sneezing, runny nose, nasal obstruction, or itchy nose, without having a cold (fever or sore throat)?"). Chronic rhinosinusitis was diagnosed when the subjective symptoms of both nasal obstruction and discharge were present for more than 3 months and an intranasal endoscopic examination 
Table 1. Prevalence of any and annoying tinnitus by gender and age group in participants over 12 years old $(n=21893)$

\begin{tabular}{|c|c|c|c|c|c|c|c|c|}
\hline \multirow{2}{*}{ Characteristics } & \multicolumn{4}{|c|}{ Any tinnitus } & \multicolumn{4}{|c|}{ Annoying tinnitus } \\
\hline & $\%^{a}$ & Prevalence & $95 \% \mathrm{Cl}$ & $P$-value & $\%^{b}$ & Prevalence & $95 \% \mathrm{Cl}$ & $P$-value \\
\hline \multicolumn{9}{|l|}{ Gender } \\
\hline Male & 44.95 & 17.73 & $16.73-18.74$ & $<0.001^{c}$ & 40.51 & 28.27 & 25.69-30.84 & $0.25^{c}$ \\
\hline Female & 55.05 & 21.66 & $20.54-22.79$ & & 59.49 & 30.16 & $27.63-32.69$ & \\
\hline \multicolumn{9}{|l|}{ Age (years) } \\
\hline Total $(\geq 12)$ & 100 & 19.70 & 18.84-20.56 & $<0.001^{d}$ & 100 & 29.31 & $27.33-31.29$ & $<0.001^{d}$ \\
\hline $12-18$ & 10.11 & 17.63 & $15.38-19.88$ & & 8.71 & 15.72 & $11.32-20.13$ & \\
\hline $19-29$ & 14.40 & 16.67 & $14.82-18.51$ & & 9.15 & 19.49 & $14.59-24.40$ & \\
\hline $30-39$ & 16.52 & 17.27 & $15.79-18.75$ & & 13.65 & 20.10 & $16.34-23.85$ & \\
\hline $40-49$ & 16.03 & 16.25 & $14.67-17.82$ & & 13.01 & 24.77 & $20.35-29.18$ & \\
\hline $50-59$ & 16.77 & 21.11 & $19.39-22.84$ & & 16.25 & 33.78 & $29.51-38.05$ & \\
\hline $60-69$ & 12.57 & 26.43 & $24.54-28.32$ & & 18.79 & 39.26 & $35.07-43.45$ & \\
\hline$>70$ & 13.60 & 32.12 & 29.94-34.30 & & 20.43 & 51.65 & $47.68-55.62$ & \\
\hline
\end{tabular}

$\mathrm{Cl}$, confidence interval.

apercent of Korean population over 12 years old.

bPercent of population with any tinnitus.

${ }^{\mathrm{c}}$ Rao-Scott Chi-square test was used.

${ }^{\mathrm{d}}$ For the age-related trend test, logistic regression analysis was used.

showed objective findings of discolored nasal drainage or polyps. $^{6}$

\section{Evaluation of associated factors}

As tests and questionnaires related to balance problems were performed only in participants aged 40 years or older, we analyzed the correlation between tinnitus and the associated factors in participants over that age. We initially selected 13419 participants aged 40 years or older. Subjects with any missing data were dropped from the association analysis, leaving only 5140 complete cases.

Potential associated factors from the basic health examination and interview were evaluated for their association with the incidence and severity of tinnitus in a total of 5140 participants (2280 men and 2860 women). Data from the otolaryngologic survey and examinations, including TM perforation, hearing loss, dizziness and vestibular dysfunction, rhinitis, and chronic rhinosinusitis were evaluated. Age, sex, marital status, household income (grouped as lower, lower middle, upper middle, and upper), quality of life (evaluated by EQ-5D score from EuroQol group ${ }^{7}$ ), smoking (past/current), drinking history (more than once a month), history of stroke, experience of depressive mood, and recognition of stress (from the questionnaire) were also included. In addition, anemia (defined as hemoglobin $<13 \mathrm{~g} / \mathrm{dL}$ for men, and $<12 \mathrm{~g} / \mathrm{dL}$ for women), diabetes (defined as fasting blood glucose level $>126 \mathrm{mg} / \mathrm{dL}$ ), hepatitis $B$ (measured by electrochemiluminescence immunoassay), and obesity (defined as body mass index $>25 \mathrm{~kg} / \mathrm{m}^{2}$ ) were included. Potentially associated factors were evaluated by univariable analysis. Only variables with a $P$-value $\leq 0.05$ were selected for multivariable analysis in the logistic regression model. Associated factors for any tinnitus were evaluated in the total population and those for annoying tinnitus were evaluated among subjects with any tinnitus.

\section{Statistical analysis}

The prevalence and 95\% confidence intervals (CIs) for any and annoying tinnitus were calculated. In univariable analysis, Rao-Scott Chi-square test (using PROC SURVEYFREQ in SAS version 9.3; SAS Institute, Cary, NC, USA) and logistic regression analysis (using PROC SURVEYLOGISTIC in SAS) were used to test the association between tinnitus and risk factors in a complex sampling design. In multivariable analysis, adjusted odds ratios (ORs) with 95\% CIs were calculated using logistic regression analysis (using PROC SURVEYLOGISTIC in SAS). To reflect national population estimates, sample weights were applied in all analyses. All $P$-values were two-sided, and $P<0.05$ was considered to be statistically significant.

\section{RESULTS}

\section{Prevalence of tinnitus}

Among the 21893 participants $\geq 12$ years of age, 4523 had experienced tinnitus in the prior 12 months; the prevalence of any tinnitus was $19.7 \%$ (95\% CI 18.8\%-20.6\%). Among 4512 participants who experienced any tinnitus, 1466 suffered from annoying tinnitus in daily life, representing 29.3\% (95\% CI $27.3 \%-31.3 \%$ ) of the tinnitus population and $5.8 \%$ of the total population. Any tinnitus and annoying tinnitus increased with age $(P<0.001)$, and prevalence of any tinnitus was higher in women than men $(P<0.001)$. However, there was no sex difference $(P=0.25)$ in presence of annoying tinnitus among those with any tinnitus (Table 1).

In a subset of 5140 participants $\geq 40$ years of age in whom analysis of associated factors was performed, the prevalence of any tinnitus was $20.7 \%$ (95\% CI $19.1 \%-22.2 \%$ ). Among participants $\geq 40$ years of age reporting any tinnitus ( $n=1112$ ), the prevalence of annoying tinnitus was $34.8 \%$ (95\% CI $31.1 \%-38.5 \%)$. 
Table 2. Analysis of factors potentially associated with any tinnitus in participants over 40 years old $(n=5140)$

\begin{tabular}{|c|c|c|c|c|c|c|c|c|}
\hline \multirow{2}{*}{ Variables } & \multirow{2}{*}{$n\left(\%^{\mathrm{a}}\right)$} & \multirow{2}{*}{ Tinnitus } & \multicolumn{3}{|c|}{ Univariable analysis } & \multicolumn{3}{|c|}{ Multivariable analysis ${ }^{c}$} \\
\hline & & & $P$-value & OR & $95 \% \mathrm{Cl}$ & $P$-value & OR & $95 \% \mathrm{Cl}$ \\
\hline \multicolumn{9}{|c|}{ Demographic characteristics } \\
\hline Age, years (mean) & & 57.23 & $<0.001$ & 1.03 & $1.02-1.04$ & $<0.001$ & 1.02 & $1.01-1.03$ \\
\hline \multicolumn{9}{|l|}{ Sex } \\
\hline Male (\%) & $2280(48.88)$ & 20.33 & & Referent & & & & \\
\hline Female (\%) & $2860(51.12)$ & 20.99 & 0.62 & 1.04 & $0.89-1.22$ & & & \\
\hline \multicolumn{9}{|l|}{ Marital status } \\
\hline No $(\%)$ & $64(1.65)$ & 16.27 & 0.42 & 0.74 & $0.36-1.53$ & & & \\
\hline Yes (\%) & $5076(98.35)$ & 20.74 & & Referent & & & & \\
\hline \multicolumn{9}{|l|}{ Income } \\
\hline Lower (\%) & $1208(25.16)$ & 20.48 & & Referent & & & & \\
\hline Lower middle (\%) & $1297(25.65)$ & 22.83 & 0.73 & 1.15 & $0.86-1.53$ & & & \\
\hline Upper middle (\%) & $1328(25.26)$ & 20.70 & 1.00 & 1.01 & $0.76-1.36$ & & & \\
\hline Upper (\%) & $1307(23.94)$ & 18.52 & 0.92 & 0.88 & $0.66-1.18$ & & & \\
\hline \multicolumn{9}{|c|}{ Otolaryngologic conditions (Physical examination and questionnaire) } \\
\hline \multicolumn{9}{|l|}{ Falling attack } \\
\hline No $(\%)$ & $5058(98.29)$ & 20.26 & & Referent & & & Referent & \\
\hline Yes (\%) & $82(1.71)$ & 44.24 & $<0.001$ & 3.13 & $1.75-5.58$ & 0.20 & 1.42 & $0.83-2.43$ \\
\hline \multicolumn{9}{|l|}{ History of dizziness } \\
\hline No $(\%)$ & $4169(82.24)$ & 17.48 & & Referent & & & Referent & \\
\hline Yes $(\%)$ & $971(17.76)$ & 35.43 & $<0.001$ & 2.59 & $2.13-3.15$ & $<0.001$ & 2.11 & $1.72-2.59$ \\
\hline \multicolumn{9}{|c|}{ Vertigo, subjective positional } \\
\hline No $(\%)$ & $5007(97.87)$ & 20.40 & & Referent & & & Referent & \\
\hline Yes $(\%)$ & $133(2.13)$ & 32.90 & $<0.01$ & 1.91 & $1.26-2.92$ & 0.15 & 0.73 & $0.47-1.13$ \\
\hline \multicolumn{9}{|l|}{ Vestibular dysfunction } \\
\hline No $(\%)$ & $4978(97.22)$ & 20.41 & & Referent & & & Referent & \\
\hline Yes $(\%)$ & $162(2.78)$ & 29.83 & 0.02 & 1.66 & $1.06-2.59$ & 0.28 & 0.75 & $0.44-1.27$ \\
\hline \multicolumn{9}{|l|}{ Cholesteatoma } \\
\hline No $(\%)$ & $5021(97.78)$ & 20.57 & & Referent & & & & \\
\hline Yes (\%) & $119(2.22)$ & 25.04 & 0.36 & 1.29 & $0.74-2.24$ & & & \\
\hline \multicolumn{9}{|l|}{ Hearing loss } \\
\hline No $(\%)$ & $4199(84.80)$ & 17.84 & & Referent & & & Referent & \\
\hline Yes (\%) & $941(15.20)$ & 36.45 & $<0.001$ & 2.64 & $2.15-3.24$ & $<0.001$ & 2.04 & $1.61-2.58$ \\
\hline \multicolumn{9}{|c|}{ Otitis media with effusion } \\
\hline No $(\%)$ & $5117(99.56)$ & 20.59 & & Referent & & & & \\
\hline Yes $(\%)$ & $23(0.44)$ & 37.08 & 0.08 & 2.27 & $0.91-5.67$ & & & \\
\hline \multicolumn{9}{|l|}{ Rhinitis } \\
\hline No $(\%)$ & $4117(79.42)$ & 19.22 & & Referent & & & Referent & \\
\hline Yes (\%) & $1023(20.58)$ & 26.26 & $<0.001$ & 1.50 & $1.22-1.83$ & $<0.001$ & 1.48 & $1.20-1.84$ \\
\hline \multicolumn{9}{|l|}{ Rhinosinusitis } \\
\hline No $(\%)$ & 4869 (94.62) & 20.28 & & Referent & & & Referent & \\
\hline Yes $(\%)$ & $271(5.38)$ & 27.47 & 0.01 & 1.49 & $1.10-2.02$ & 0.23 & 1.21 & $0.89-1.66$ \\
\hline \multicolumn{9}{|l|}{ TM perforation } \\
\hline No $(\%)$ & 4984 (97.01) & 20.13 & & Referent & & & Referent & \\
\hline Yes (\%) & $156(2.99)$ & 38.07 & $<0.001$ & 2.44 & $1.58-3.77$ & 0.09 & 1.51 & $0.94-2.44$ \\
\hline
\end{tabular}

\section{Analysis of associated factors}

Experiences of any/annoying tinnitus and associated factors were investigated using univariable and multivariable analyses. In univariable analysis, any tinnitus was associated with increased stress, depressive mood, history of stroke, angina, and better quality of life. Among the otolaryngologic problems, TM perforation, hearing loss, rhinosinusitis, and balance problems (occurrence of dizziness or imbalance, subjective positional vertigo, falling attack, and vestibular dysfunction) were also associated with any tinnitus.

After multivariable-adjusted analysis, older age (OR 1.02, $95 \%$ CI $1.01-1.03$ ), better quality of life (OR $0.99,95 \%$ CI
0.99-1.00), hearing loss (OR 2.04, 95\% CI 1.61-2.58), rhinitis (OR 1.48, 95\% CI 1.20-1.84), depressive mood (OR 1.56, 95\% CI 1.14-2.15), and experiencing dizziness or imbalance (OR 2.11, 95\% CI 1.72-2.59) remained as associated factors of any tinnitus (Table 2). For annoying tinnitus, stress, diabetes, obesity, history of cardiac disease, hearing loss, and vestibular dysfunction were associated in univariable analysis. After multivariable-adjusted analysis, older age (OR 1.03, 95\% CI 1.01-1.04), hearing loss (OR $1.55,95 \%$ CI 1.09-2.21), history of cardiac disease (OR 5.51, 95\% CI 1.04-29.20), and stress (OR 1.64, 95\% CI 1.14-2.36) were associated with annoying tinnitus (Table 3). 


\begin{tabular}{|c|c|c|c|c|c|c|c|c|}
\hline \multirow{2}{*}{ Variables } & \multirow{2}{*}{$n(\% \mathrm{a})$} & \multirow{2}{*}{ Tinnitus } & \multicolumn{3}{|c|}{ Univariable analysis } & \multicolumn{3}{|c|}{ Multivariable analysis $^{c}$} \\
\hline & & & $P$-value & OR & $95 \% \mathrm{Cl}$ & $P$-value & OR & $95 \% \mathrm{Cl}$ \\
\hline \multicolumn{9}{|c|}{ Data obtained from the questionnaire } \\
\hline \multicolumn{9}{|c|}{ Alcohol drinking } \\
\hline No $(\%)$ & $1630(27.60)$ & 22.93 & \multicolumn{3}{|c|}{ Referent } & \multicolumn{3}{|c|}{ Referent } \\
\hline Yes (\%) & $3510(72.40)$ & 19.80 & 0.05 & 0.83 & $0.69-1.00$ & 0.40 & 1.09 & $0.90-1.31$ \\
\hline \multicolumn{9}{|l|}{ Depressive mood } \\
\hline No $(\%)$ & 4909 (95.99) & 20.17 & \multicolumn{3}{|c|}{ Referent } & \multicolumn{3}{|c|}{ Referent } \\
\hline Yes $(\%)$ & $231(4.01)$ & 32.57 & $<0.001$ & 1.91 & $1.42-2.58$ & 0.01 & 1.56 & $1.14-2.15$ \\
\hline \multicolumn{9}{|l|}{ History of anxiety } \\
\hline No $(\%)$ & $5075(98.93)$ & 20.56 & \multicolumn{3}{|c|}{ Referent } & & & \\
\hline Yes (\%) & $65(1.07)$ & 30.48 & 0.12 & 1.70 & $0.87-3.30$ & & & \\
\hline \multicolumn{9}{|l|}{ History of cardiac disease } \\
\hline No $(\%)$ & $5015(98.07)$ & 20.48 & \multicolumn{3}{|c|}{ Referent } & \multicolumn{3}{|c|}{ Referent } \\
\hline Yes (\%) & $125(1.93)$ & 30.28 & 0.02 & 1.69 & $1.07-2.66$ & 0.31 & 1.27 & $0.80-2.01$ \\
\hline \multicolumn{9}{|l|}{ History of stroke } \\
\hline No (\%) & $5032(98.46)$ & 20.48 & \multicolumn{3}{|c|}{ Referent } & \multicolumn{3}{|c|}{ Referent } \\
\hline Yes (\%) & $108(1.54)$ & 32.76 & $<0.01$ & 1.89 & $1.22-2.93$ & 0.35 & 1.29 & $0.76-2.20$ \\
\hline Smoking, current & & & & & & & & \\
\hline No $(\%)$ & $4156(76.66)$ & 20.92 & & Referent & & & & \\
\hline Yes (\%) & $984(23.34)$ & 19.84 & 0.54 & 0.94 & $0.76-1.16$ & & & \\
\hline Smoking, past & & & & & & & & \\
\hline No $(\%)$ & $3049(55.06)$ & 20.82 & & Referent & & & & \\
\hline Yes (\%) & $2091(44.94)$ & 20.48 & 0.81 & 0.98 & $0.83-1.16$ & & & \\
\hline Stress & & & & & & & & \\
\hline No $(\%)$ & 3805 (73.52) & 19.55 & & Referent & & & Referent & \\
\hline Yes (\%) & $1335(26.48)$ & 23.78 & $<0.01$ & 1.28 & $1.08-1.53$ & 0.17 & 1.15 & $0.94-1.39$ \\
\hline Visual disturbance & & & & & & & & \\
\hline No $(\%)$ & $5098(99.38)$ & 20.63 & & Referent & & & & \\
\hline Yes $(\%)$ & $42(0.62)$ & 26.45 & 0.40 & 1.38 & $0.65-2.96$ & & & \\
\hline Quality of life score (mean) ${ }^{b}$ & & 70.05 & $<0.001$ & 0.99 & $0.98-0.99$ & 0.01 & 0.99 & $0.99-1.00$ \\
\hline Laboratory data & & & & & & & & \\
\hline Anemia & & & & & & & & \\
\hline No $(\%)$ & 4651 (91.51) & 20.49 & & Referent & & & & \\
\hline Yes (\%) & $489(8.47)$ & 22.58 & 0.31 & 1.13 & $0.90-1.44$ & & & \\
\hline Diabetes & & & & & & & & \\
\hline No $(\%)$ & $4459(87.31)$ & 20.38 & & Referent & & & & \\
\hline Yes (\%) & $681(12.69)$ & 22.64 & 0.32 & 1.14 & $0.88-1.49$ & & & \\
\hline Hepatitis B & & & & & & & & \\
\hline No $(\%)$ & 4957 (96.31) & 20.80 & & Referent & & & & \\
\hline Yes (\%) & $183(3.69)$ & 79.20 & 0.31 & 0.80 & $0.52-1.23$ & & & \\
\hline Hypercholesterolemia & & & & & & & & \\
\hline No $(\%)$ & $4298(83.88)$ & 20.36 & & Referent & & & & \\
\hline Yes (\%) & $842(16.12)$ & 22.25 & 0.28 & 1.12 & $0.91-1.37$ & & & \\
\hline Hypertension & & & & & & & & \\
\hline No $(\%)$ & 3204 (64.63) & 19.83 & & Referent & & & & \\
\hline Yes (\%) & 1936 (35.37) & 22.20 & 0.09 & 1.15 & $0.98-1.36$ & & & \\
\hline Obesity & & & & & & & & \\
\hline No $(\%)$ & 3319 (63.88) & 20.93 & & Referent & & & & \\
\hline Yes (\%) & $1821(36.12)$ & 20.21 & 0.63 & 0.96 & $0.80-1.14$ & & & \\
\hline
\end{tabular}

$\mathrm{Cl}$, confidence interval; OR, odds ratio; TM, tympanic membrane.

apercent of Korean population aged $\geq 40$ years.

${ }^{\mathrm{b}}$ Continuous variables were denoted by mean. In the non-tinnitus group, the mean age was 53.68 , and the mean quality of life score was 74.80 . 'Variables with $P$-value $\leq 0.05$ in univariable analysis were selected.

Univariable and multivariable analyses were done using the logistic regression model.

\section{DISCUSSION}

This is the first epidemiologic study to investigate the prevalence and associated factors of tinnitus based on representative data from a government-centered survey in South Korea. Accurate epidemiologic information may contribute to the proper provision of health care, preventive screenings, and rehabilitative services.

Tinnitus is the perception of sound from the ears or head without an audible external source and is known to be more common among older adults. ${ }^{8}$ The severity of tinnitus ranges from barely noticeable (any tinnitus) to debilitating (annoying 
Table 3. Analysis of potentially associated factors with 'annoying tinnitus' in participants over 40 years old $(n=1112)$

\begin{tabular}{|c|c|c|c|c|c|c|c|c|}
\hline \multirow{2}{*}{ Variables } & \multirow{2}{*}{$\%^{a}$} & \multirow{2}{*}{$\begin{array}{l}\text { Annoying } \\
\text { tinnitus }\end{array}$} & \multicolumn{3}{|c|}{ Univariable analysis } & \multicolumn{3}{|c|}{ Multivariable analysis $^{c}$} \\
\hline & & & $P$-value & OR & $95 \% \mathrm{Cl}$ & $P$-value & OR & $95 \% \mathrm{Cl}$ \\
\hline \multicolumn{9}{|c|}{ Demographic characteristics } \\
\hline Age, years $(\text { mean })^{\mathrm{b}}$ & & 60.10 & $<0.0001$ & 1.04 & $1.02-1.05$ & 0.0007 & 1.03 & $1.01-1.04$ \\
\hline \multicolumn{9}{|l|}{ Sex } \\
\hline Male (\%) & $491(48.09)$ & 34.44 & & Referent & & & & \\
\hline Female (\%) & $621(51.91)$ & 35.12 & 0.83 & 1.03 & $0.78-1.36$ & & & \\
\hline \multicolumn{9}{|l|}{ Marital status } \\
\hline No $(\%)$ & $13(1.30)$ & 45.46 & 0.50 & 1.57 & $0.43-5.77$ & & & \\
\hline Yes $(\%)$ & 1099 (98.70) & 34.65 & & Referent & & & & \\
\hline \multicolumn{9}{|l|}{ Income } \\
\hline Lower (\%) & $263(24.93)$ & 36.42 & & Referent & & & & \\
\hline Lower middle (\%) & $301(28.33)$ & 35.37 & 1.00 & 0.96 & $0.61-1.50$ & & & \\
\hline Upper middle (\%) & $273(25.29)$ & 35.41 & 1.00 & 0.96 & $0.58-1.57$ & & & \\
\hline Upper (\%) & $275(21.45)$ & 31.42 & 0.81 & 0.80 & $0.49-1.30$ & & & \\
\hline \multicolumn{9}{|c|}{ Otolaryngologic conditions (Physical examination and questionnaire) } \\
\hline \multicolumn{9}{|l|}{ Falling attack } \\
\hline No $(\%)$ & $1074(96.34)$ & 34.67 & & Referent & & & & \\
\hline Yes (\%) & $38(3.66)$ & 38.02 & 0.72 & 1.16 & $0.52-2.56$ & & & \\
\hline \multicolumn{9}{|l|}{ History of dizziness } \\
\hline No $(\%)$ & 764 (69.56) & 33.48 & & Referent & & & & \\
\hline Yes (\%) & $348(30.44)$ & 37.80 & 0.29 & 1.21 & $0.85-1.71$ & & & \\
\hline \multicolumn{9}{|c|}{ Vertigo, subjective positional } \\
\hline No $(\%)$ & $1066(96.60)$ & 34.27 & & Referent & & & & \\
\hline Yes $(\%)$ & $46(3.40)$ & 49.52 & 0.11 & 1.88 & $0.87-4.06$ & & & \\
\hline \multicolumn{9}{|l|}{ Vestibular dysfunction } \\
\hline No $(\%)$ & $1064(95.99)$ & 34.08 & & Referent & & & Referent & \\
\hline Yes $(\%)$ & $48(4.01)$ & 51.84 & 0.02 & 2.08 & $1.10-3.93$ & 0.56 & 1.27 & $0.57-2.83$ \\
\hline \multicolumn{9}{|l|}{ Cholesteatoma } \\
\hline No (\%) & $1080(97.31)$ & 34.87 & & Referent & & & & \\
\hline Yes $(\%)$ & $32(2.69)$ & 32.14 & 0.79 & 0.89 & $0.36-2.19$ & & & \\
\hline \multicolumn{9}{|l|}{ Hearing loss } \\
\hline No (\%) & 768 (73.19) & 30.25 & & Referent & & & Referent & \\
\hline Yes (\%) & $344(26.80)$ & 47.18 & $<0.001$ & 2.06 & $1.52-2.80$ & 0.01 & 1.55 & $1.09-2.21$ \\
\hline \multicolumn{9}{|c|}{ Otitis media with effusion } \\
\hline No $(\%)$ & $1100(99.21)$ & 34.65 & & Referent & & & & \\
\hline Yes (\%) & $12(0.79)$ & 53.26 & 0.29 & 2.15 & $0.52-8.86$ & & & \\
\hline \multicolumn{9}{|l|}{ Rhinitis } \\
\hline No (\%) & $836(73.85)$ & 34.52 & & Referent & & & & \\
\hline Yes (\%) & $276(26.15)$ & 35.56 & 0.80 & 1.05 & $0.73-1.51$ & & & \\
\hline \multicolumn{9}{|l|}{ Rhinosinusitis } \\
\hline No (\%) & 1037 (92.86) & 34.36 & & Referent & & & & \\
\hline Yes (\%) & $75(7.14)$ & 40.45 & 0.38 & 1.30 & $0.73-2.31$ & & & \\
\hline \multicolumn{9}{|l|}{ TM perforation } \\
\hline No (\%) & $1056(94.50)$ & 34.07 & & Referent & & & & \\
\hline Yes (\%) & $56(5.50)$ & 47.19 & 0.08 & 1.73 & $0.94-3.17$ & & & \\
\hline
\end{tabular}

tinnitus). Patients who experience tinnitus often report significant associated morbidities such as lifestyle detriment, emotional difficulties, sleep deprivation, work hindrance, and interference with social interaction. ${ }^{4}$

Several population-based studies have attempted to estimate the prevalence of tinnitus, and the reported prevalence from each region varies partly due to the different characteristics of the surveyed population or definition of tinnitus, such as duration, frequency, and severity. In a questionnaire-based survey conducted in England in 1978, the prevalence of subjective tinnitus was $15.5 \%-18.6 \%$, and the prevalence of annoying tinnitus was $0.4 \%-2.8 \%$ in respondents over 17 years of age. ${ }^{9}$ A 1989 study from Sweden and a 1996 study from Italy reported the prevalence of subjective tinnitus as $14.2 \%$ and $14.5 \%$, respectively. ${ }^{10,11}$ Data from the National Health Interview Survey in 1996 revealed that tinnitus was experienced by 35-50 million adults in the US, and 2-3 million reported symptoms that were severely debilitating. ${ }^{12}$ The overall prevalence of tinnitus in Egypt was 5.17\%, and the highest age-specific prevalence was $17.7 \%$, among subjects above 60 years of age. $^{3}$ With regard to Asian countries, epidemiologic studies from Japan $^{1}$ in a population aged 65 years or older and from China ${ }^{13}$ in subjects over 10 years of age reported that the overall prevalence of tinnitus was $18.6 \%$ and $14.5 \%$, respectively. In the 2004 NHANES, 


\begin{tabular}{|c|c|c|c|c|c|c|c|c|}
\hline \multirow{2}{*}{ Variables } & \multirow{2}{*}{$\%^{a}$} & \multirow{2}{*}{$\begin{array}{c}\text { Annoying } \\
\text { tinnitus }\end{array}$} & \multicolumn{3}{|c|}{ Univariable analysis } & \multicolumn{3}{|c|}{ Multivariable analysis ${ }^{c}$} \\
\hline & & & $P$-value & OR & $95 \% \mathrm{Cl}$ & $P$-value & OR & $95 \% \mathrm{Cl}$ \\
\hline \multicolumn{9}{|c|}{ Data obtained from the questionnaire } \\
\hline \multicolumn{9}{|c|}{ Alcohol drinking } \\
\hline No $(\%)$ & $390(30.62)$ & 38.53 & & Referent & & & & \\
\hline Yes $(\%)$ & $722(69.38)$ & 33.15 & 0.11 & 0.79 & $0.59-1.05$ & & & \\
\hline \multicolumn{9}{|l|}{ Depressive mood } \\
\hline No $(\%)$ & $1034(93.67)$ & 34.34 & & Referent & & & & \\
\hline Yes $(\%)$ & $78(6.33)$ & 41.52 & 0.25 & 1.36 & $0.80-2.29$ & & & \\
\hline \multicolumn{9}{|l|}{ History of anxiety } \\
\hline No $(\%)$ & $1091(98.42)$ & 34.9 & & Referent & & & & \\
\hline Yes (\%) & $21(1.58)$ & 30.91 & 0.75 & 0.84 & $0.28-2.49$ & & & \\
\hline \multicolumn{9}{|l|}{ History of cardiac disease } \\
\hline No $(\%)$ & $1103(99.48)$ & 34.54 & & Referent & & & Referent & \\
\hline Yes (\%) & $9(0.52)$ & 83.08 & 0.008 & 9.28 & $1.79-48.13$ & 0.04 & 5.51 & $1.04-29.20$ \\
\hline \multicolumn{9}{|l|}{ History of stroke } \\
\hline No (\%) & $1079(97.56)$ & 34.51 & & Referent & & & & \\
\hline Yes (\%) & $33(2.44)$ & 46.04 & 0.28 & 1.62 & $0.68-3.88$ & & & \\
\hline \multicolumn{9}{|l|}{ Smoking, current } \\
\hline No $(\%)$ & 919 (77.59) & 35.19 & & Referent & & & & \\
\hline Yes (\%) & $193(22.41)$ & 33.43 & 0.69 & 0.93 & $0.63-1.36$ & & & \\
\hline \multicolumn{9}{|l|}{ Smoking, past } \\
\hline No $(\%)$ & $661(55.46)$ & 34.28 & & Referent & & & & \\
\hline Yes (\%) & $451(44.54)$ & 35.44 & 0.74 & 1.05 & $0.78-1.43$ & & & \\
\hline \multicolumn{9}{|l|}{ Stress } \\
\hline No $(\%)$ & $772(69.54)$ & 31.95 & & Referent & & & Referent & \\
\hline Yes (\%) & $340(30.46)$ & 41.29 & 0.02 & 1.50 & $1.07-2.09$ & $<0.01$ & 1.64 & $1.14-2.36$ \\
\hline \multicolumn{9}{|l|}{ Visual disturbance } \\
\hline No $(\%)$ & $1100(99.21)$ & 34.71 & & Referent & & & & \\
\hline Yes (\%) & $12(0.79)$ & 44.76 & 0.51 & 1.52 & $0.43-5.40$ & & & \\
\hline Quality of life score (mean) ${ }^{b}$ & & 68.16 & 0.06 & 0.99 & $0.98-1.00$ & & & \\
\hline \multicolumn{9}{|l|}{ Laboratory data } \\
\hline \multicolumn{9}{|l|}{ Anemia } \\
\hline No $(\%)$ & $997(90.73)$ & 34.85 & & Referent & & & & \\
\hline Yes (\%) & $115(9.27)$ & 34.20 & 0.90 & 0.97 & $0.62-1.53$ & & & \\
\hline \multicolumn{9}{|l|}{ Diabetes } \\
\hline No $(\%)$ & $954(86.10)$ & 33.43 & & Referent & & & Referent & \\
\hline Yes (\%) & $158(13.90)$ & 43.22 & 0.03 & 1.52 & $1.03-2.24$ & 0.26 & 1.25 & $0.85-1.84$ \\
\hline \multicolumn{9}{|l|}{ Hepatitis B } \\
\hline No $(\%)$ & 1077 (96.91) & 35.01 & & Referent & & & & \\
\hline Yes (\%) & $35(3.09)$ & 27.91 & 0.44 & 0.72 & $0.31-1.65$ & & & \\
\hline \multicolumn{9}{|l|}{ Hypercholesterolemia } \\
\hline No $(\%)$ & $924(82.65)$ & 33.58 & & Referent & & & & \\
\hline Yes (\%) & $188(17.35)$ & 40.57 & 0.16 & 1.35 & $0.89-2.05$ & & & \\
\hline Hypertension & & & & & & & & \\
\hline No $(\%)$ & $660(62.02)$ & 32.58 & & Referent & & & & \\
\hline Yes $(\%)$ & $452(37.98)$ & 38.41 & 0.07 & 1.29 & $0.98-1.70$ & & & \\
\hline Obesity & & & & & & & & \\
\hline No (\%) & 719 (64.68) & 37.11 & & Referent & & & Referent & \\
\hline Yes $(\%)$ & $393(35.32)$ & 30.56 & 0.05 & 0.75 & $0.56-1.00$ & 0.06 & 0.75 & $0.56-1.01$ \\
\hline
\end{tabular}

$\mathrm{Cl}$, confidence interval; OR, odds ratio; TM, tympanic membrane.

apercent of Korean population aged $\geq 40$ years.

${ }^{\mathrm{b} C}$ Continuous variables were denoted by mean. In the non-annoying tinnitus group, the mean age was 55.70 , and the mean of quality of life score was 71.06 .

cVariables with $P$-value $\leq 0.05$ in univariable analysis were selected.

Univariable and multivariable analyses were done using the logistic regression model.

a cross-sectional tinnitus study of 14178 participants in the US, the prevalence of experiencing any tinnitus and frequent tinnitus was $25.3 \%$ and $7.9 \%$, respectively. The prevalence of any and frequent tinnitus increased with age, peaking at $31.4 \%$ and $14.3 \%$, respectively, at $60-69$ years of age (Table 4). ${ }^{4}$ In the present study, the prevalence of any tinnitus was $19.7 \%$, and that of annoying tinnitus was $5.8 \%$ in those over 12 years of age.

In a US population-based longitudinal study conducted in Beaver Dam, Wisconsin, the 5-year incidence of tinnitus among those free of tinnitus at baseline was $5.7 \%$, and the 10 -year cumulative incidence of tinnitus was $12.7 \%$, in 2922 
Table 4. Prevalence of tinnitus in previous reports

\begin{tabular}{|c|c|c|c|c|}
\hline Authors & Year & Sample & $\begin{array}{l}\text { Prevalence of } \\
\text { any tinnitus }\end{array}$ & $\begin{array}{l}\text { Prevalence of } \\
\text { severe tinnitus }\end{array}$ \\
\hline MRCIHR ${ }^{9}$ & 1981 & England, 19000 persons aged over 17 years & $17.0 \%$ & $8.0 \%$ \\
\hline Axelsson et $\mathrm{al}^{10}$ & 1989 & Sweden, 2378 persons aged $20-80$ years & $14.2 \%$ & $2.4 \%$ \\
\hline Quaranta et al $^{11}$ & 1996 & Italy, 2170 persons aged over 18 years & $14.5 \%$ & \\
\hline Shargorodsky et $\mathrm{al}^{4}$ & 2010 & USA, 1999-2004 NHANES, 14178 persons aged over 20 years & $25.3 \%$ & $7.9 \%$ \\
\hline Michikawa et al ${ }^{1}$ & 2010 & Japan, 1320 persons aged over 65 years & $18.6 \%$ & \\
\hline Kehdr et $\mathrm{al}^{3}$ & 2010 & Egypt, 8484 persons aged over 6 years & $5.17 \%$ & $0.8 \%$ \\
\hline Xu et $\mathrm{al}^{13}$ & 2011 & China, 6333 persons aged over 10 years & $14.5 \%$ & \\
\hline Fuji et $\mathrm{al}^{32}$ & 2011 & Japan, 14423 persons aged $45-79$ years & $11.9 \%$ & $0.4 \%$ \\
\hline This study & & South Korea, 21893 persons aged over 12 years & $19.7 \%$ & $5.8 \%$ \\
\hline
\end{tabular}

MRCIHR, Medical Research Council's Institute of Hearing Research; NHANES, National Health and Nutrition Examination Survey.

participants aged 48 to 92 years. Risk factors for occurrence of tinnitus included hearing loss, high serum total cholesterol, history of head injury, otosclerosis, arthritis, and history of smoking. ${ }^{2,8}$

A clear relationship has been established between hearing loss and tinnitus, and the majority of tinnitus patients have some degree of hearing loss. Since hearing loss and tinnitus are so closely related, populations with more prevalent hearing loss have a corresponding greater prevalence of tinnitus. ${ }^{14}$ In a report from Egypt in 2010, 53\% of tinnitus patients reported hearing loss. ${ }^{3}$ In a report from China, ${ }^{13}$ the prevalence of tinnitus was $30.0 \%$ and $6.0 \%$, respectively, among people with and without hearing impairment. In the latter study, there was a significant relationship between high-frequency hearing impairment ( $>25 \mathrm{~dB} \mathrm{HL}$ at $4 \mathrm{kHz}$ ) and high-pitched tinnitus (OR 6.61). History of middle ear infections and noise exposure were also risk factors (OR 5.90 and 2.74, respectively). In the 1999-2004 NHANES, frequent tinnitus was also associated with low-mid-frequency (OR 2.37, 95\% CI 1.76-3.21) and high-frequency (OR 3.00, 95\% CI 1.78-5.04) hearing impairment. ${ }^{4}$ Kim et al reported that, even if patients with tinnitus do not have any subjective hearing impairment, most of them have high-frequency hearing loss (above $2 \mathrm{kHz}$ ) and/or extended-high-frequency hearing loss (above $8 \mathrm{kHz}$ ). ${ }^{15}$ Our results also confirm that unilateral or bilateral hearing loss ( $>25 \mathrm{~dB}$ HL) was associated with any tinnitus (OR 2.04) and annoying tinnitus (OR 1.55).

Regarding the relationship between tinnitus and vestibular dysfunction, our results indicate that any tinnitus, but not annoying tinnitus, was associated with experience of dizziness or imbalance (OR 2.11, 95\% CI 1.72-2.59). The Blue Mountains Hearing Study also reported dizziness as a prognostic factor for incident tinnitus. ${ }^{16}$ Although the mechanism behind this association is still unclear, a possible explanation is that the dysfunction of the vestibular labyrinth might have an association with the auditory pathways that causes tinnitus.

The prevalence of hearing loss and tinnitus increases with age. About $27 \%$ of all individuals between the ages of 60 and 69 years and $45 \%$ of those persons 70 years and older have subjective hearing loss in South Korea. ${ }^{17}$ The 1999-2004
NHANES data have established that the odds of any tinnitus (OR 1.67, 95\% CI 1.44-1.94) and frequent tinnitus (OR 5.43, 95\% CI 3.93-7.50) appear to peak at 60-69 years of age compared with subject under 50-years-of-age. ${ }^{4}$ Although hearing loss is commonly associated with tinnitus in older patients, other medical conditions become increasingly prevalent in this population and must be considered as potential causes of tinnitus. These conditions include vascular disease, middle-ear disease, diabetes, hypertension, autoimmune disorders, and degenerative neural disorders with or without concomitant hearing loss. ${ }^{14,18,19}$

Differences in the prevalence of tinnitus according to gender have been amply demonstrated. The US National Center for Health Statistics in 1996 reported that prevalence of tinnitus was $12 \%$ for men and $7 \%$ for women over 65 years of age. ${ }^{12}$ The 1999-2004 NHANES demonstrated that prevalence of any tinnitus for men and women was $26.1 \%$ and $24.6 \%$, respectively, in participants over the age of 20 years, and that this difference was significant. ${ }^{4}$ In our study, prevalence of any tinnitus was $17.7 \%$ for men and $21.7 \%$ for women $(P<0.001)$ in participants over the age of 12 years, and this tendency was consistent through the surveyed period. Similarly, the prevalence of annoying tinnitus was $28.3 \%$ for men and $30.2 \%$ for women among those with any tinnitus $(P=0.25)$. These results might be attributed to a more stressful cultural situation for women in South Korea, which demands obedience and more roles in the family. A survey from Japan also demonstrated that tinnitus was more prevalent in women than in men, though without statistical significance. ${ }^{1}$

The neurophysiologic model of tinnitus postulates that tinnitus emerges as a result of the interaction of a number of neural subsystems. The auditory pathways plays a role in the perception of tinnitus, whereas the limbic system is responsible for the development of tinnitus annoyance. Therefore, psychological stress, anxiety, and depressive mood can be associated with the development and severity of tinnitus. ${ }^{20-23}$ Epidemiologic studies have demonstrated that stress, anxiety, depressive mood, and sleep deprivation were associated with tinnitus, ${ }^{24}$ and $36 \%-62 \%$ of tinnitus patients were found to be depressed in previous studies. ${ }^{25,26}$ 
In the 2011 Beaver Dam offspring study, depressive symptoms (OR 2.04, 95\% CI 1.50-2.76) and the use of anti-depressant medications (OR 1.58, 95\% CI 1.17-2.14) were significantly associated with tinnitus. ${ }^{27}$ The 1999-2004 NHANES also demonstrated the association of generalized anxiety and major depressive disorders with tinnitus among the 2265 participants between the ages of 20 and 39 years of age. After multivariable adjustment, participants with generalized anxiety disorder had higher multivariate-adjusted odds of any tinnitus (OR 2.66, 95\% CI 1.32-5.34) and frequent tinnitus (OR 6.07, 95\% CI 2.33-15.78) than those without anxiety disorder. As for depressive disorder, only the odds of any tinnitus remained significant after adjustment (OR 2.01, 95\% CI 1.24-3.25). ${ }^{4}$ Epidemiologic studies from Japan in a population over 65 years of age ${ }^{1}$ and from Egypt in the general population over 6 years of age $^{3}$ also showed that depressive mood and emotional stress were associated with tinnitus. Our results also demonstrated that occurrence of any tinnitus is associated with depressive mood (OR 1.56, 95\% CI 1.14-2.15), and annoying tinnitus with stress (OR 1.64, 95\% CI 1.14-2.36).

Tinnitus can result in sleep deprivation, decreased work productivity, and overall lifestyle detriment, ${ }^{26,28,29}$ which consequently leads to psychological distress and depression. ${ }^{30}$ Likewise, major depressive and generalized anxiety disorders may exacerbate tinnitus, while their treatment might alleviate tinnitus. ${ }^{31}$ Depression and anxiety scores have been reported to be higher at the early stage of tinnitus development. ${ }^{20}$

In our survey, history of cardiac disease has a strong association with annoying tinnitus (OR 5.51, 95\% CI 1.04-29.20). This association has been reported in several studies, ${ }^{1,11,32}$ but the mechanism is not clear. The vascular dysregulation hypothesis is one possible theory for the development of tinnitus. ${ }^{33}$ Dysregulation is often associated with a reduced cardiac output, as well as with reflex activation of vasoconstrictive systems, including the sympathetic nervous system and renin-angiotensin-aldosterone system. ${ }^{33}$ The results of the present study support the hypothesis that cardiovascular disorders may be associated with severe tinnitus.

As this study utilized cross-sectional data from annual surveys, we were unable to obtain incidence estimates and risk factors. Instead, we were able present only prevalence rates and associated factors of tinnitus, which is a limitation of this study. Another limitation of the study is that relatively few participants were included in the association analysis. A large number of cases were dropped due to missing data and age restriction of the vestibular survey, which only included participants with complete data aged 40 years or older. Among a total of 13419 participants aged 40 years or older, 5140 had a complete data set. As participants in the association subset were not significantly different from the total participants in the prevalence of any/annoying tinnitus and male to female ratio, important characteristics of the population who were enrolled in the association analysis were not largely changed by exclusion of the missing data.

In summary, the overall prevalence of any tinnitus is $19.7 \%$, and the prevalence of annoying tinnitus is $5.8 \%$ in the general population of South Korea. Tinnitus is more prevalent in women than in men, and the prevalence increases with age. Analysis of associated factors demonstrated that any tinnitus is associated with better quality of life, hearing loss, rhinitis, depressive mood, and feelings of dizziness or imbalance. Annoying tinnitus is associated with hearing loss, history of cardiac disease, and stress.

This study demonstrates that tinnitus is a common condition and that a large population suffers from annoying tinnitus in South Korea. Public acknowledgement and further intervention to modify factors associated with tinnitus are required for prevention and management of the condition, for which no known cure exists.

\section{ACKNOWLEDGMENTS}

We thank the 150 residents of the otorhinolaryngology departments of 47 training hospitals in South Korea and members of the Division of Chronic Disease Surveillance in Korea Centers for Disease Control \& Prevention for participating in this survey and the dedicated work they provided.

Conflicts of interest: None declared.

\section{REFERENCES}

1. Michikawa T, Nishiwaki Y, Kikuchi Y, Saito H, Mizutari K, Okamoto $\mathrm{M}$, et al. Prevalence and factors associated with tinnitus: a community-based study of Japanese elders. J Epidemiol. 2010;20:271-6.

2. Nondahl DM, Cruickshanks KJ, Wiley TL, Klein BE, Klein R, Chappell R, et al. The ten-year incidence of tinnitus among older adults. Int J Audiol. 2010;49:580-5.

3. Khedr EM, Ahmed MA, Shawky OA, Mohamed ES, El Attar GS, Mohammad KA. Epidemiological study of chronic tinnitus in Assiut, Egypt. Neuroepidemiology. 2010;35:45-52.

4. Shargorodsky J, Curhan GC, Farwell WR. Prevalence and characteristics of tinnitus among US adults. Am J Med. 2010; 123:711-8.

5. American Academy of Otolaryngology-Head and Neck Surgery Ffoundation I. Committee on Hearing and Equilibrium guidelines for the evaluation of results of treatment of conductive hearing loss. Otolaryngol Head Neck Surg. 1995; 113:186-7.

6. Benninger MS, Ferguson BJ, Hadley JA, Hamilos DL, Jacobs M, Kennedy DW, et al. Adult chronic rhinosinusitis: definitions, diagnosis, epidemiology, and pathophysiology. Otolaryngol Head Neck Surg. 2003;129(3 Suppl):S1-32.

7. Hurst NP, Kind P, Ruta D, Hunter M, Stubbings A. Measuring health-related quality of life in rheumatoid arthritis: validity, responsiveness and reliability of EuroQol (EQ-5D). Br J Rheumatol. 1997;36:551-9. 
8. Nondahl DM, Cruickshanks KJ, Wiley TL, Klein R, Klein BE, Tweed TS. Prevalence and 5-year incidence of tinnitus among older adults: the epidemiology of hearing loss study. J Am Acad Audiol. 2002;13:323-31.

9. Epidemiology of tinnitus. Medical Research Council's Institute of Hearing Research. Ciba Found Symp. 1981;85:16-34.

10. Axelsson A, Ringdahl A. Tinnitus - a study of its prevalence and characteristics. Br J Audiol. 1989;23:53-62.

11. Quaranta A, Assennato G, Sallustio V. Epidemiology of hearing problems among adults in Italy. Scand Audiol Suppl. 1996;42: 9-13.

12. Adams PF, Hendershot GE, Marano MA; Centers for Disease Control and Prevention/National Center for Health Statistics. Current estimates from the National Health Interview Survey, 1996. Vital Health Stat 10. 1999;(200):1-203.

13. Xu X, Bu X, Zhou L, Xing G, Liu C, Wang D. An epidemiologic study of tinnitus in a population in Jiangsu Province, China. J Am Acad Audiol. 2011;22:578-85.

14. Henry JA, Dennis KC, Schechter MA. General review of tinnitus: prevalence, mechanisms, effects, and management. J Speech Lang Hear Res. 2005;48:1204-35.

15. Kim DK, Park SN, Kim HM, Son HR, Kim NG, Park KH, et al. Prevalence and significance of high-frequency hearing loss in subjectively normal-hearing patients with tinnitus. Ann Otol Rhinol Laryngol. 2011;120:523-8.

16. Gopinath B, McMahon CM, Rochtchina E, Karpa MJ, Mitchell P. Risk factors and impacts of incident tinnitus in older adults. Ann Epidemiol. 2010;20:129-35.

17. Cho YS, Choi SH, Park KH, Park HJ, Kim JW, Moon IJ, et al. Prevalence of otolaryngologic diseases in South Korea: data from the Korea national health and nutrition examination survey 2008. Clin Exp Otorhinolaryngol. 2010;3:183-93.

18. Meikle MB. Electronic access to tinnitus data: the Oregon Tinnitus Data Archive. Otolaryngol Head Neck Surg. 1997;117: 698-700.

19. Ahmad N, Seidman M. Tinnitus in the older adult: epidemiology, pathophysiology and treatment options. Drugs Aging. 2004;21: 297-305.

20. Park SN, Park DS, Park KH, Kim JH, Han MA, Yeo SW.
Measurement of stress, anxiety and depression in the patients with tinnitus and their clinical significance. Korean J Audiol. 2007;11:22-8.

21. Baguley D. Neurophysiological approach to tinnitus patients. Am J Otol. 1997;18:265-6.

22. Jastreboff PJ, Hazell JW. A neurophysiological approach to tinnitus: clinical implications. Br J Audiol. 1993;27:7-17.

23. Jastreboff PJ. Phantom auditory perception (tinnitus): mechanisms of generation and perception. Neurosci Res. 1990;8: 221-54.

24. Vesterager V. Combined psychological and prosthetic management of tinnitus: a cross-sectional study of patients with severe tinnitus. Br J Audiol. 1994;28:1-11.

25. Harrop-Griffiths J, Katon W, Dobie R, Sakai C, Russo J. Chronic tinnitus: association with psychiatric diagnoses. J Psychosom Res. 1987;31:613-21.

26. Tyler RS, Baker LJ. Difficulties experienced by tinnitus sufferers. J Speech Hear Disord. 1983;48:150-4.

27. Nondahl DM, Cruickshanks KJ, Huang GH, Klein BE, Klein R, Nieto FJ, et al. Tinnitus and its risk factors in the Beaver Dam offspring study. Int J Audiol. 2011;50:313-20.

28. Folmer RL, Griest SE. Tinnitus and insomnia. Am J Otolaryngol. 2000;21:287-93.

29. Folmer RL, Griest SE, Meikle MB, Martin WH. Tinnitus severity, loudness, and depression. Otolaryngol Head Neck Surg. 1999;121:48-51.

30. Crocetti A, Forti S, Ambrosetti U, Bo LD. Questionnaires to evaluate anxiety and depressive levels in tinnitus patients. Otolaryngol Head Neck Surg. 2009;140:403-5.

31. Folmer RL, Shi YB. SSRI use by tinnitus patients: interactions between depression and tinnitus severity. Ear Nose Throat J. 2004;83:107-8, 10, 12 passim.

32. Fujii K, Nagata C, Nakamura K, Kawachi T, Takatsuka N, Oba $\mathrm{S}$, et al. Prevalence of tinnitus in community-dwelling Japanese adults. J Epidemiol. 2011;21:299-304.

33. Ramani GV, Uber PA, Mehra MR. Chronic heart failure: contemporary diagnosis and management. Mayo Clin Proc. 2010;85:180-95. 